\title{
La Ventosa de Malmström en Sicoprofilaxis Obstétrica*
}

Por los Dres.: Isaías Arenas Buenahora, Enrique Sánchez Puyana

y Gregorio Mantilla Cadena

Del Equipo Iván Pávlov de Bucaramanga

\section{CAPITULO I}

\section{HISTORIA - DESCRIPCION DEL APARATO}

Hace aproximadamente 250 años se están haciendo ensayos tendientes a reducir el trabajo del parto y los esfuerzos maternos durante el mismo por medio de tracciones aplicadas a la cabeza del feto. Así vemos que el fórceps fué inventado por la Familia Chamberlin a principios del Siglo XVII, y muchas variedades de este instrumento están actualmente en uso en las maternidades dol mundo entero.

El uso del vacío como medio para fijar un elemento de traccićn a la cabeza fetal fué ensayado por el inglés Yonge, quien utilizó una ventosa en el año de 1706. En el año de 1849 Sinpson hizo el segundo ensayo de esta índole utilizando una cúpula de caucho conectada a una jeringa de latón. En 1890 Mac Cahey, con los mismos fines, hizo uso de una cúpula de caucho provista de una manija firme que se oprimía sobre la presentación creando su propio vacío.

Torpín en 1938 reporta 10 partos con trabajo prolongado, en los cuales aplicó una cúpula de succión hecha de caucho, en la que se podía hacer el vacío con una bomba provista de una manija de tracción y orejas que permitían al operador hacer la rotación. En 1947 Couzigou, describe por primera vez una cúpula metálica que llamó ventosa, nombre utilizado en los países de habla fran-

* Trabajo presentado al III Siniposio Colombiano de Sicoprofilaxis Obstétrica. - Julio de 1961 - Bucaramanga, Colombia. 
cesa. Koller en 1950 aplicaba en la atonía uterina una tracción prolongada hasta de 18 horas por medio de un aparato de succión con un vacío de 0.5 a $1.5 \mathrm{kgs}$. provocando con esto una activación de la contracción. Gastaldo en 1951 usó también una cúpula metálica cubierta de caucho. Finderle en 1952 descubrió su cúpula metálica en forma de trompeta cuyo vacío se hacía por medio de una bomba; más tarde anunciaba que su instrumento había reemplazado al fórceps completamente durante tres años.

Malmstrom ideó su primer instrumento de succión con una copa en parte hecha de caucho, que no llenó todos los requerimientos ni dio todos los resultados deseados como un verdadero instrumento de parto. Solamente en 1956, cuando ideó su nuevo modelo, que es el que hemos usado para la elaboración de este trabajo, se tuvo un verdadero instrumento de marcada efectividad, poniéndose en uso desde su primer reporte en la mayor parte de los países europeos incluyendo Rusia, donde Petchenko lo empleó en el año de 1958: también en Africa del Norte, Africa Central, China y América del Sur.

En Norte América películas demostrativas de su uso fueron exhibidas por Muller y Watteville en el Segundo Congreso Mundial de Obstetricia y Ginecología reunido en Montreal en 1958. Las únicas referencias bibliográficas británicas encontradas, fueron las publicaciones pediátricas de Lobban en 1955 y el artículo publicado en el British Medical Journal del 4 de junio de 1960 por los doctores Chaimers y Fothergill, que reportan sus experiencias en 100 casos de aplicación de Vacuum-Extractor.

De su aplicación en Colombia tenemos noticia de que en el Hospital de la Hortúa de Bogotá, en el año de 1952 aproximadamente, se trabajó con un aparato de fabricación nacional que funcionaba al vacío, cuyos resultados ignoramos, habiéndonos sido imposible encontrar alguna publicación al respecto.

Consultando la bibliografía mundial, hemos comprobado que no se ha hecho hasta ahora ninguna publicación de su uso en sicoprofilaxis.

\section{DECRIPCION DEL APARATO}

El instrumento consta de tres campanas de metal con una profundidad de $20 \mathrm{~mm}$. v diámetros de 40, 50 y $60 \mathrm{~mm}$; en el fondo de estas cúpulas va colocada una placa de metal de cuyo 
centro parte una cadena de tracción que pasa a través de un tubo de aspiración de goma, formando así un tubo portacadena, que termina en un mango de metal, llamado mango de tracción, en donde se asegura la cadena con una aguja que atraviesa dicho mango. De ahí parte otro tubo de caucho llamado tubo del vacío, que se conecta a un frasco de vidrio, botella de vacío, cerrada por un tapón de goma perforado por tres partes: una para el manómetro de vacío graduado en décimas de kilogramo por centímetro cuadrado de superficie, y las otras dos ocupadas con dos conectores metálicos, uno para el tubo que va a la campana y el otro a la bomba de aspiración. Sobre este último hay un tornillo válvula para suspender o disminuír el vacío.

Las campanas mayores llevan un botón utilizado para la rotación o como indicador de ésta.

La botella de vacío va colocada dentro de una canastilla fabricada de alambre y plástico provista de una gotera para colocar la bomba y un gancho en su parte superior que sirve para suspender el aparato (ver dibujo).

\section{CAPITULO II}

\section{MECANISMO DE ACCION - TECNICA DE APLICACION}

$\mathrm{Su}$ fundamento es el de provocar un vacío que, partiendo del frasco de vidrio, se transmita por los tubos de caucho a la campana metálica y tỉje ésta al cuero cabelludo, para obtener así una forma de tracción sobre la cabeza fetal tendiente a reducir el trabajo de parto.

Al poner en íntimo contacto la campana con el cuero cabelludo y provocar el vacío, se produce un edema parecido al del caput sucedaneum normal que viene a llenar completamente la campana, tomando su forma y fijando el aparato para ejercer posteriormente las tracciones. En el momento de éstas se produce un estiramiento del cuero cabelludo que trae como consecuencia, indirectamente, una reducción de los diámetros cefálicos ocasionada por las fuerzas descompuestas que obran en todos los sentidos sobre la cabeza fetal.

Siendo este un aparato flexible, las tracciones imprimirán a la cabeza los distintos movimientos que debe seguir para reco- 
rrer fisiológicamente el canal del parto, disminuyendo en esta forma la morbilidad materno-fetal.

Finalmente, durante el desprendimiento, el operador puede controlar la dilatación del anillo vulvar logrando con esto una mejor protección del periné.

Técnica de aplicación. - La esterilización del aparato se hace por ebullición, al autoclave o por inmersión en substancias antisépticas. Nosotros preferimos este último procedimiento y lo hacemos en Neo-vack durante 10 minutos.

Antes de proceder a su aplicación es necesario hacer un cuidadoso examen de la paciente para precisar el estado del canal vaginal, el grado de dilatación del cuello, estado de las membranas, altura, variedad y grado de flexión de la presentación.

$\mathrm{Su}$ aplicación es posible desde los cuatro centímetros de dilatación, espacio que permite el paso de la ventosa más pequeña, y a la altura del primero, segundo y tercer planos de la pelvis o sea aplicación alta, media y baja.

Montado el aparato en la forma anteriormente descrita, es necesario hacer una purga del mismo aplicando la campana contra la mano del operador y haciendo varias succiones para cerciorarse de la permeabilidad de los tubos. Enseguida se usa un lubricante (aceite, glicerina o jabón líquido) para facilitar la introducción de la ventosa. Nosotros acostumbramos introducir la campana en un poco de agua tibia para darle la temperatura adecuada y no impresionar a la paciente, ya que en su aplicación no usamos ninguna anestesia. Aconsejamos hacer una previa limpieza del moco contenido en la vagina para evitar obstrucciones en los tubos. Luego procedemos a la introducción de la campana elegida, deprimiendo el periné bien sea con los dedos índice y medio de la mano izquierda, o con el borde de la misma campana que se introduce lateralmente pegada a la pared vaginal posterior, para rotarla más arriba y adaptarla al vértex, teniendo el cuidado de no dejar incluído el borde del cuello, las paredes vaginales ni las fontanelas. Cuando se usan las campanas provistas de botón, éste debe estar dirigido hacia el punto de referencia de la presentación para que sirva de indicador de la rotación.

En los casos de las presentaciones de vértice mal flejadas, variedades posteriores, se debe procurar colocar la campana lo 
más posteriormente posible para que al hacer la tracción se corrija este ligera deflexión.

Después de fijado el aparato, y cuando la ventosa hace contacto perfectamente con el cuero cabelludo, se inicia el vacío con $0.2 \mathrm{kgs}$; ; este vacío se debe aumentar poco a poco, una décima cada dos minutos hasta llegar a 0.6 para tracción simple y 0.8 si se quiere corregir deflexiones o imprimir rotación artificial. La existencia de un caput sucedaneum espontáneo, prominente, favorece la aplicación de la ventosa y permite una presa rápida, provocando este vacío de una sola vez.

Logrado este tiempo, se inician las tracciones que deben hacerse con la mano derecha colocada en el mango de tracción y los decos índice y medio de la izquierda en el borde superior de la campana. El movimiento de tracción ejercido con la mano derecha debe ser simultáneo con la presión dirigida hacia el sacro con los dedos de la mano izquierda, a fin de que la cabeza siga la dirección del canal genital. A este movimiento de tracción se le puede imprimir un movimiento circular que es efectivo en muchos casos.

Es importante hacer las tracciones durante las contracciones uterinas o el pujo para aprovechar la fuerza de éstos y no correr el riesgo de que se suelte el aparato.

En muchos casos la rotación espontánea de la cabeza es debida a la interacción de las fuerzas del parto y las fuerzas de tracción. La rotación artıficial de la presentación puede ser necesaria en algunos casos. Según Malmstrom, ésta puede llevarse a cabo con el dedo pulgar de la mano izquierda y con la ayuda del botón de rotación, movimiento que debe ser simultáneo con la tracción.

Nosotros creemos que no es el aparato ideal para imprimir esta rotación artificial, pues en muchos casos no la hemos logrado y entonces preferimos continuar haciendo una tracción adecuada con lo cual la cabeza rota espontáneamente.

Al chocar la cabeza contra el periné, y al iniciarse la dilatación del anillo vulvar, acostumbramos para la tracción cambiar la mano derecha por la izquierda colocada sobre la superficie externa de la campana y con la mano libre proteger el periné, obteniendo así un control perfecto de la dilatación de éste y del desprendimiento lento y progresivo de la cabeza. Una vez realizado 
el parto del primer polo se afloja la válvula para eliminar el vacío y soltar el aparato.

El caput-sucedaneum artificial es inevitable por la naturaleza misma del método (ver ilustraciones).

\section{CAPITULO III}

\section{INDICACIONES}

En un principio estaba indicado para la tracción prolongada y continua, pero más recientemente ha sido usado de manera creciente para la extracción directa del feto.

En numerosas clínicas ha reemplazado al fórceps pero varios autores piensan que debía utilizarse más como ayuda suplementaria que como sustituto de aquél.

$1^{0}$ - Inserción baja de la placenta y placenta previa marginal con el fin de fijar la presentación, cohibir la hemorragia y acelerar los períodos del parto.

$2^{\circ}$ - Desprendimiento prematuro de la placenta normo-inserta, cuando el estado del cuello y las condiciones generales de la madre lo permitan.

$3^{o}$ - Inercia uterina secundaria, donde obraría como estimulante de la contracción y al mismo tiempo como aparato ex. tractor.

$4^{c}$ - Distocia de encajamiento de causa secundaria: Brevedad relativa del cordón, ligera deflexión de la cabeza, intensidad insuficiente de la contracción, etc.

$5^{\text {o }}$ - Detención o perversión de la rotación; obraría como un rotador fisiológico de la presentación, ayudado por las fuerzas naturales de la madre.

$6^{0}$ - Estenosis pélvica relativa; como prueba de encajamiento ya que no aumenta los diámetros cefálicos, logrando en algunos casos el parto por vías naturales.

$7^{\circ}$ - Cicatrices uterinas: cesáreas anteriores, miomectomías, etc., para abreviar el período expulsivo.

$8^{\circ}$ - Presentaciones de vértice y sincipucio con flexión defectuosa, como aparato flexor. 
$9^{\circ}$ - En los casos de período expulsivo prolongado.

$10^{\circ}$ - Sufrimiento fetal no agudo.

$11^{\circ}$ - Pre-eclampsia y eclampsia, para acelerar el parto cuando las condiciones de! cuello lo permitan.

$12^{\circ}$ - Enfermedades cardíacas y pulmonares, para ahorrar los esfuerzos del período expulsivo.

$13^{\circ}$ - Presentaciones de pelvis.

$14^{0}$ - En casos especiales de presentaciones de frente, pelvis muy amplia y feto pequeño (un caso en nuestro trabajo en el primer feto de un embarazo gemelar).

$15^{\circ}$ - En sicoprofilaxis como "fórceps de complacencia" para abreviar el trabajo en las primíparas, usándolo en el período expulsivo, ya que el cansancio es una de las causas más frecuentes del descontrol que originan el dolor.

$16^{\circ}$ - No estamos de acuerdo con algunos autores en preconizar su uso en casos de prematuridad, pues consideramos que cualquier maniobra instrumental se sumaría al problema de inmadurez ya existente. Acostumbramos usarlo por encima del octavo mes de embarazo en caso necesario.

\section{CAPITULO IV \\ EXPERIENCIA PERSONAL}

Efectos sobre la madre. - Su uso ofrece el máximo de seguridad para la madre, por las siguientes razones:

a) El riesgo de sepsis es mínimo ya que el instrumento no penetra en la parte alta del canal genital.

b) No hay aumento de los diámetros cefálicos, como sucede con el fórceps, y por lo tanto todo el espacio utilizable de la pelvis queda libre para el paso del feto, no produciéndose por lo tanto ningún traumatismo en el canal del parto.

c) No se causa daño a la madre ni al niño, dado que la ventosa se desprende cuando se ejercen esfuerzos excesivos y mal dirigidos.

d) No hemos tenido ni ha sido reportado en la literatura ninguna complicación vesical. 
e) Hay menos peligros de desgarros perineales debido a que con el mismo aparato se controla fácilmente el desprendimiento.

f) No hay peligro de rupturas uterinas por las mismas razones expuestas anteriormiente.

En los diez meses comprendidos entre Agosto de 1960 y Junio de 1961, utilizamos la ventosa en 38 pacientes, con los siguientes resultados:

\section{CUADRO No 1}

Resultados según paridad

Paridad

Para I

Para IV

Para VI

Para X

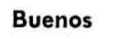

15

\section{1}

1
Regulares

19

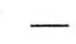$$
-
$$$$
-
$$

Malos No de casos

De este cuadro podemos deducir que de los 38 casos, 3 eran multíparas, 35 primíparas y que solamente hubo un fracaso.

\section{CUADRO No 2}

Resultados según el grado de dilatación
Grado de dilatación

Completa

9 centímetros

8 centímetros

Buenos Regulares

17

1
16

1

2
Malos

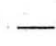

1
35

1

1

Como vemos, el mayor número de casos se aplicó con dilatación completa.

\section{CUADRO No 3}

Resultados según altura de la presentación

Plano de aplıcación

Primer plano

Segundo plano

Tercer plano

$\begin{array}{cccc}\text { Buenos } & \text { Regulares } & \text { Malos } & \text { No de casos } \\ 2 & 2 & - & 4 \\ 5 & 9 & 1 & 15 \\ 11 & 8 & - & 19\end{array}$


CUADRO No 4

Resultados según variedad de posición

Variedad de posición

O. I. A.

O. I. T.

O. I. P.

O. D. A.

O. D. T.

O. D. P.

N. I. A.

\begin{tabular}{r} 
Buenos \\
5 \\
1 \\
\hline 1 \\
1 \\
10
\end{tabular}

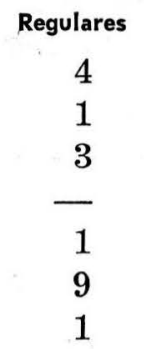

Malos

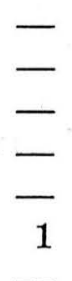

$\mathbf{N}^{\circ}$ de casos

9

2

3

1

2

20

1

La mayor incidencia de aplicación la tuvimos en variedad D. P. que es la que ocasiona expulsivos prolongados. El único caso que figura de presentación de frente fué un caso especial de un primer feto en embarazo gemelar y parto prematuro con pelvis materna muy amplia.

$$
\text { CUADRO No } 5
$$

Resultados según indicación

Indicación

Profiláctico

Expulsivo prolongado

Distocia de encajam.

Pelvis oblicua

Present. frente

(1er. feto gemelar)

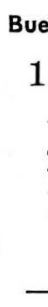

\begin{abstract}
Regulares
\end{abstract}
14

Malos

11

4

2

1

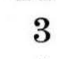

1

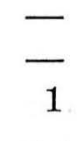

1
No de casos

25

7

4

1

La mayor parte de las aplicaciones se hicieron profilácticamente. Los 4 casos de distocia de encajamiento tuvieron como causa: Inercia uterina, 2 casos. Inserción baja de placenta 1, y brevedad relativa del cordón 1 (2 circulares).

$$
\text { CUADRO } \mathrm{N}^{\circ} 6
$$

Resultados según diámetro de la campana

Diámetro

Pequeña

Mediana

Grande

$\begin{array}{cc}\text { Buenos } & \text { Regulares } \\ 1 & 3 \\ 16 & 18 \\ 4 & 1\end{array}$

Malos

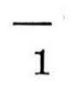

4

35

5

En la mayor parte de los casos se usó la campana mediana. 


$$
\text { CUADRO No } 7
$$

Analgesia medicamentosa

Con analgesia

$$
\text { Buenos Regulares }
$$

Sin analgesia

$\begin{array}{rccc}1 & \text { Regulares } & \text { Malos } & \text { No de casos } \\ 17 & 1 & 1 & 3 \\ & 18 & - & 35\end{array}$

CUADRO N⿳ 8

Resultados según tiempo de aplicación

$\begin{array}{rcccc}\text { Tiempo en minutos } & \text { Buenos } & \text { Regulares } & \text { Malos } & \text { No de casos } \\ 5 \text { a } 15 & 5 & 3 & - & 8 \\ 16 \text { a } 25 & 6 & 6 & - & 12 \\ 26 \text { a } 35 & 6 & 5 & 1 & 12 \\ 36 \text { a } 45 & - & 3 & - & 3 \\ 46 \text { a } 55 & - & 2 & - & 2 \\ 56 \text { a más de } 1 \text { hora } & 1 & - & - & 1\end{array}$

Podemos ver que el tiempo promedio de aplicación del aparato es de 16 a 35 minutos, teniendo en cuenta que se gastan de 8 a 10 minutos en la fijación de la campana.

$$
\text { CUADRO No } 9
$$

Resultados según duración del expulsivo

Tiempo en minutos

20 a 40

41 a 60

61 a 80

81 a 100

$\begin{array}{cc}\text { Buenos } & \text { Regulares } \\ 7 & 9 \\ 8 & 7 \\ 2 & 2 \\ 1 & 1\end{array}$

El mayor número de casos tuvo un expulsivo comprendido entre 20 y 60 minutos pero vale la pena anotar que la campana se aplicó en un altísimo porcentaje después de varios minutos de iniciado este período.

$$
\text { CUADRO No } 10
$$

Resultados según peso del niño

$\begin{array}{cccc}\text { Buenos } & \text { Regulares } & \text { Malos } & \text { No de casos } \\ 2 & 3 & 1 & 6 \\ 5 & 6 & - & 11 \\ 10 & 7 & - & 17 \\ 1 & 3 & - & 4\end{array}$


Hacemos notar que no hubo fracasos del método en las series de mayores pesos fetales.

Efectos sobre el niño. - La experiencia ha comprobado la inocuidad de este instruniento sobre el feto, por las siguientes razones :

a) Se ha demostradc matemáticamente que la compresión ejercida sobre la cabeza fetal, es inferior a la vigésima parte de la ejercida en los partos intervenidos con fórceps.

b) Por la razón anterior, por no existir un aumento de los diámetros cefálicos y por no poder abusar de la fuerza en la tracción, lógicamente el traumatismo craneano es mínimo.

c) Se elimina la posibilidad de parálisis, cicatrices faciales y lesiones oculares.

d) La presencia del caput artificial es inevitable por la naturaleza misma del método.

$\mathrm{Al}$ desprender la vertosa de la cabeza fetal se observa una importante prominencia que disminuye rápidamente en tamaño y baja casi completamente en pocas horas para quedar convertida en una bolsa clásica.

Pequeñas equuímosis y aún ligeras erosiones circinadas pueden persistir durante varios días, pero desaparecen sin causar ningún disturbio. Algunos autores anotan necrosis del cuero cabelludo que desaparecen fácilmente pero en nuestra casuística no hemos tenido ningún caso.

Goldberg y Levy (1958) anotan que la única complicación fetal posible es la formación de un hematoma que fué evitado con la aplicación de vitaminas $\mathrm{K}$ y un vendaje compresivo. Muller indica que estas medidas se consideran como innecesarias; en caso de formación de este hematoma siguiendo una conducta apropiada, se puede asegurar que no queda ningún daño permanente en el cuero cabelludo.

En el estudio que se presenta se controlaron 38 recién nacidos cuyos datos más importantes se resumen así: 
Vol. XIII

No 1

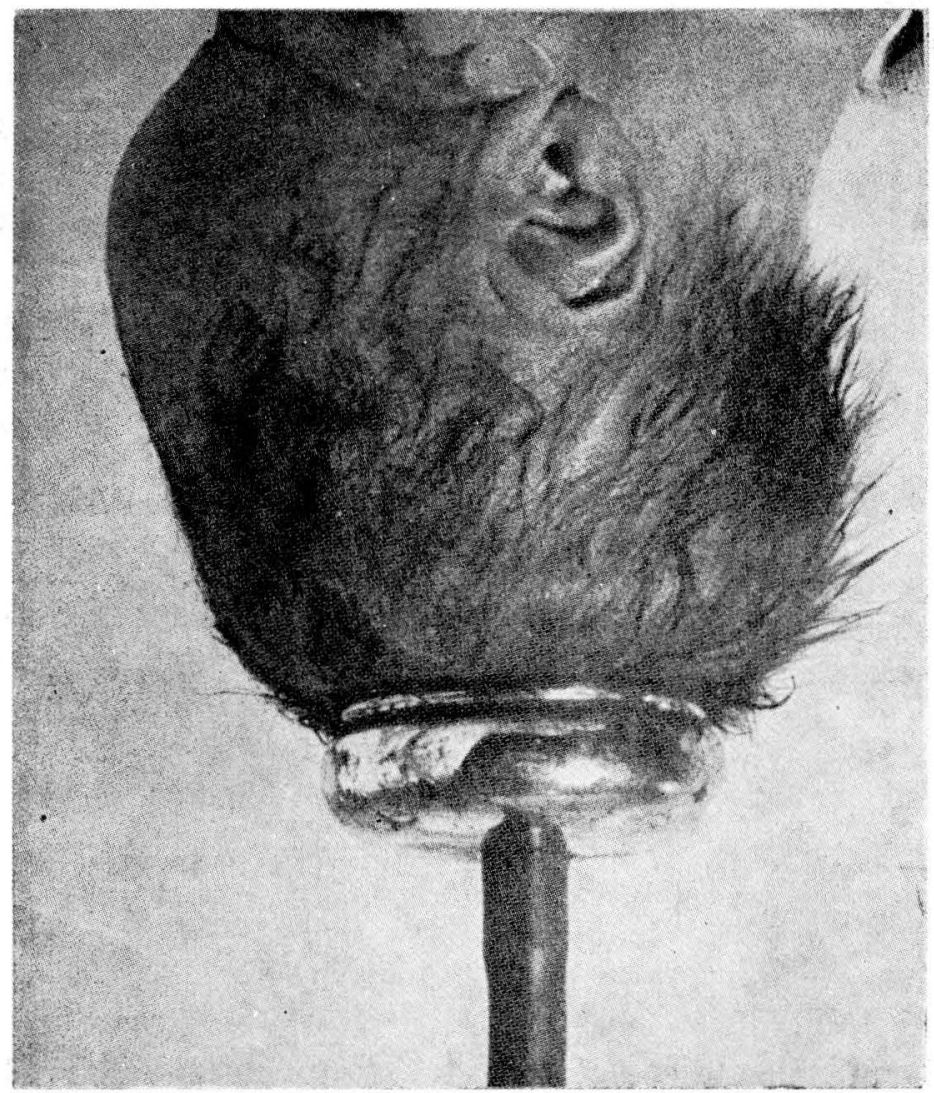

\section{CUADRO No 11}

Características generales

$\begin{array}{llllllllllllllll}\text { Niños } & \ldots & \ldots & \ldots & \ldots & \ldots & \ldots & 20 & \text { Niñas } & \ldots & \ldots & \ldots & \ldots & \ldots & \ldots & 18\end{array}$

Peso mínimo . . . 2.500 grs. Peso máximo . . . 4.500 grs.

Mayor frecuencia de peso entre 3.000 a 4.000 grs.

Mayor frecuencia de talla 50 cts. 


\section{CUADRO No 12 \\ Particularidades de las bosas}

Pequeñas

Medianas

Grandes

Excoriaciones

Edema

Sepsis
10 casos

20 casos

8 casos

5 casos

3 casos

2 casos

\section{Reabsorciones:}

Pequeñas

3 días

Medianas

5 días

Grandes

15 días

El mayor número de casos fue de bosas medianas. El tiempo de reabsorción no solo está condicionado a su tamaño sino también a la presencia de excoriaciones, edema y sepsis sobreagregados a las mismas.

Los dos casos de sepsis cedieron en 3 días.

La localización de las bosas fué de predominancia parieto-occipital izquierda o derecha. Es importante anotar que en ninguno de los casos quedaron incluídas las fontanelas dentro de las bosas.

\section{CUADRO No 13}

Evaluación del recién nacido

$\begin{array}{lr}\text { Apgar } & 10 \\ \text { Apgar } & 9 \\ \text { Apgar } & 8 \\ \text { Apgar } & 7\end{array}$

31 casos

2 casos

4 casos

1 caso

Como podemos observar, en un alto número de casos la evaluación del recién nacido por el sistema de Apgar fue de 10.

Los dos casos de Apgar 9 tuvieron como causa obstrucción por flemas de las vías respiratorias altas.

Los cuatro casos de Apgar 8, dos tuvieron como causa obstrucción por flemas; uno, síndrome de adaptación y otro traumatismo del parto ocasionado por el uso de un fórceps laborioso complementario a la ventosa y de anestesia por gases.

El caso de Apgar 7 lo ocasionó un trabajo prolongado en primípara añosa con sufrimiento fetal.

En la totalidad de los casos hemos podido controlar el desarrollo físico y mental de esos niños hasta la época actual, sin en- 
contrar manifestaciones patológicas en los mismos, incluyendo los Apgares calificados por debajo de 10 .

Finalmente estamos en capacidad de asegurar el beneficio y la inocuidad del aparato no solo para la madre sino para el niño desde todo punto de vista, impresión que está acorde con la bibliografía mundial al respecto.

\section{CONCLUSIONES}

1 - Consultando la bibliografía mundial hemos comprobado que no se ha hecho hasta ahora ninguna publicación sobre el uso de la Ventosa en Sicoproïilaxis Obstétrica y que en Colombia no se ha publicado ningún trabajo sobre la Ventosa Obstétrica de Malmstrom.

2 - Estamos de acuerdo con algunos autores en que puede sustituír al fórceps en muchos casos, pero no en una forma absoluta, ya que éste tiene indicaciones tan precisas que lo hacen actualmente irreemplazable por ningún otro instrumento. Consideramos que no es un aparato ideal para imprimir rotación artificial; preferimos ejercer tracciones adecuadas para que la interacción de las fuerzas del parto y las de la tracción impriman la rotación.

3 - Presentamos una estadística de 38 casos de aplicación de la Ventosa en Sicoprofilaxis.

4 - En 35 casos no usamos ninguna anestesia y en 3, anestesia general.

5 - En cuanto a la calificación se refiere, 18 casos fueron buenos, 19 regulares y un fracaso. En todos los casos la paciente ignoró la aplicación del aparato; se le informó posteriormente al respecto.

6 - Se encontraron 20 bosas medianas, 10 pequeñas y 8 grandes. En ninguno de los casos se comprometieron las fontanelas.

7 - En 5 casos hubo excoriaciones del cuero cabelludo y en 3 edema del mismo. Se presentaron 2 casos de discreta sepsis.

8 - Después de diez meses de control, ninguno de los niños 
ha acusado manifestaciones patológicas en su desarrollo físico y mental.

9 - En vista de la facilidad de su aplicación, de sus múltiples y útiles indicaciones, de su bondad para la madre y el niño, creemos que su uso debe generalizarse en todos los Servicios de Sicoprofilaxis Obstétrica y Obstetricia en general.

\section{BIBLIOGRAFIA}

1 J. A. CHAIMERS y R. J. FOTHERGILL. "Uso del Vacuum Extractor en Obstetricia". The British Medical Journal. 4 jun. 1960. Vol. I, páginas 1684-1689.

2 FRANCISCO CODECIDO y GONZALEZ PALACIOS. "Primeras Impresiones sobre el nuevo modelo de ventosa cbstétrica en Vacuum Extractor". Revista Centro Médico. (Caracas) 1959 2, 104-112.

3 FINDERLE, V. "Extractor instead of forceps". Am. J. Obst. \& Gynec., 69, 1148, 1955.

4 MALMSTROM, T. "Vacuum extractor an obstetrical instrument". Acta Obs. Et Ginec. Scandinav., 33. suppl. 4, 1954.

5 SIMPSON, J. Y. "The air tractor. As a substitute for the forceps in tedious labours". The Obstetric Memoirs and contributions, I, 498. 1855.

6 TORPIN. R. "Preliminary report of obstetric device". J. M. A. Georgia. 27, 96, 1938.

7 R. R. L. NESBITT, Jr. "Selected abstracts: Malmstrom: The vacuum extractor". American Journal of Obst. \&x Gynec. 75, 1366, 1958.

8 BRUNIQUEL, G. et ISRAEL, A. 'Contribution a I'etude de la ventouse. Son champ d'application, ses avantages". Gynecologie et Obstetrique, 57, 222 9, 1958. OTRAS PUBLICACIONES QUE PUEDEN CONSULTARSE

1200 observations de légagemerıt assisté par l'emploi du vacuum extractor au cours de I'accouchement eutocique chez les primipares. Charrier, I. et. Bull. Fel. Soc. Gyn. et Obst. 9, 33, 1967.

2 La Ventouse Eutocique. Cousigou, Y. Avenir Méd. March 49-42, 1652.

3 Cephalic traction by rubber suction cup in uterine atony. Koller, O. Acta Obst. et Gynec. scand. 30, 145, 1950. suppl. 7.

4 The Malmstrom Vacuum Exiractor. A. Swedish Obstetric Instrument. Lobban, M. Nursing Mirror, May 20, 1955.

5 Atmospherine Tractor. A new instrument and some new theories in obstetrics. Mc. Cahey, P. Med. and surg. Reporter 63, 6191890.

6 The Vacuum Extractor. Malmstrom, T. Modwives Chronicle and Nursing Notes, 70, $184,1957$.

7 Analyse de 100 cas de ventouses. Rosa, P. \& Piraux, P. Bull. Soc. Belge de Gynec. et d'obst., 27, 30, 195;

8 Defence de l'extraction par ventose. Rosa, P. Bull. Soc. Belge de Gynec. et Obstet. $25,142,1955$.

9 Practique de I'extraction par ventouses a la Maternité de Géneve. Voegeli, H. Gynec. et Obstet. 57, $95104,1958$.

10 O Vacuum Extractor em Obstetricia. Beltrao Jr. F. \& Ferreira, A. D. Arquivos Brasileiros de Medicina Nava., 18, 6267. 90, 1957.

11 Experience with Malmstrom's Vacuum Extractor. A Clinical study of 100 cases. Berggren. O. G. A. Acta Obst. et Gynec. scanlinav., 38, 315321959.

12 Intracranial haemorrhage and blood-brain barrier problems in the new-born. Gron,toft, O. Acta path. et microbiol. Scandinav., suppl. 100, 24, 1954. 